Services for adults with intellectual disability are still relatively undeveloped. Vocational training and job placement services are hard to find, even in metropolitan areas. There is some potential for the employment of people with intellectual disability in rural, agriculturally based economic activities, but this arrangement needs further exploration and consolidation.

\section{Conclusions}

Currently in India, there is a mosaic of services for people with intellectual disability. Within recent years innovative approaches for the better management of these conditions in childhood have emerged, including parents' associations, family-focused interventions and CBR programmes. Families continue to be a major source of care, but they are in need of many additional services and support. Landmark legislation to recognise and address the problem has been passed but it still requires full implementation. We anticipate a stronger, rights-based approach to service development in the years to come.

\section{References}

Girimaji, S. R. (2003) Family focused intervention in mental retardation Indian models. In Changing Marital and Family Systems: Challenges to Conventional Models in Mental Health (eds R. S. Bhatti, M. Varghese \& A. Raguram), pp. 192-199. National Institute of Mental Health and Neuro Science.

Girimaji, S. C. \& Srinath, S. (2010) Perspectives of intellectual disability in India: epidemiology, policy, services for children and adults. Current Opinion in Psychiatry, 23, 441-446.

Girimaji, S. C., Srinath, S., Seshadri, S. P., et al (1999) Family Interview for Stress and Coping in Mental Retardation (FISC-MR): a tool to study stress and coping in families of children with mental retardation. Indian Journal of Psychiatry, 41, 341-349.

Narayan, J. (2007) Intellectual disabilities in India. Presented at the Second International Conference on Intellectual Disabilities/Mental Retardation, Bangkok, 16 February. See http://www.bangkok-id-conference.org/pdf/intellectual-disabilities-in-india (accessed March 2011).

Russell, P. S. S., John, J. K. \& Lakshmanan, J. L. (1999) Family intervention for intellectually disabled children. Randomised controlled trial. British Journal of Psychiatry, 174, 254-258.

Srinath, S. \& Girimaji, S. R. (1999) Epidemiology of child and adolescent mental health problems and mental retardation. NIMHANS Journal, 17, 355-366.

World Health Organization (2007) Atlas of Global Resources for Persons with Intellectual Disabilities. WHO.

\title{
Intellectual disability and Aboriginal Australians - training needs for psychiatrists
}

\author{
Meera Roy ${ }^{1}$ and Sivasankaran Balaratnasingam ${ }^{2}$ \\ ${ }^{1}$ Consultant in Psychiatry of Learning Disability, South Birmingham Primary Care Trust, UK, email Meera.Roy@sbpct.nhs.uk; \\ ${ }^{2}$ Assistant Professor and Regional Psychiatrist, Kimberley Mental Health and Drug Service, Broome, Western Australia
}

\begin{abstract}
boriginal Australians have relatively high rates of intellectual disability, a situation that is probably due mainly to poor health and social disadvantage. Populations with high rates of intellectual disability are more at risk of developmental disorders and mental ill health. We explore the training needs for psychiatrists working with indigenous people and how they can be met.

Aboriginal Australians are the original inhabitants of the Australian continent and nearby islands. Together with the Torres Strait Islanders, they form $2.7 \%$ of the population. Aboriginal communities are diverse, with different languages and cultural practices. Historically, they were a semi-nomadic hunter-gatherer people. Each clan possessed specific territory, defined by geographical features such as rivers and mountains. They shared an intimate understanding of and relationship with the land, and that relationship was
\end{abstract}

underpinned by 'dreamtime' stories, a set of beliefs that is fundamental to their well-being. An Aboriginal person attains status by acquisition of knowledge (especially about the land) rather than by acquiring material possessions. Traditional Aboriginal society is structured by social and family systems, which organise all aspects of life and perception.

The British colonisation of Australia was a catastrophic event for the Aboriginal people. Disease, displacement and direct violence from the settlers reduced the Aboriginal population by $90 \%$ between 1788 and 1900. The settlers also introduced alcohol, and alcohol misuse played a major role in further destroying Aboriginal social structures. Between 1869 and 1969 Aboriginal children were removed from their parents by federal and state agencies and church organisations, and brought up within a 'white' culture, on such disparate grounds as child protection and maintenance of 
racial purity. These children constituted the 'stolen generation' and their institutional upbringing further destroyed historical Aboriginal social structures.

Aboriginal people's health-related beliefs are interconnected with aspects of their life such as the land, kinship obligations and religion. Well-being is contingent on the effective discharge of a person's obligations to society and the land itself (Morgan et al, 1997). Supernatural agents are regarded as an important cause of illness and to many Aboriginal peoples they provide an explanatory model of illness.

\section{Mental ill health}

Aboriginal people have significantly worse health outcomes than other Australians and their life expectancy is, on average, 17 years shorter. They continue to experience greater poverty. In the Aboriginal population, indices of social deprivation are higher - such as overcrowding, illiteracy, representation in prisons and substantiated child protection notifications - as are reports of being victims of crime (Swan \& Raphael, 1995; Cohen, 1999; Australian Health Ministers' Advisory Council, 2006). Mental ill health is also a major issue. Aboriginal rates of depression, suicide and self-harm, trauma and grief, domestic violence and substance misuse are relatively high (Swan \& Raphael, 1995; Parker, 2010). The 2004-05 National Aboriginal and Torres Strait Islander Health Survey (Australian Bureau of Statistics, 2006) found substantial psychological distress, especially among Aboriginal young people. The latter suffer more emotional and behavioural problems but are less likely than other Australians of their age to access mental health services (Zubrick et al, 2005).

The prevalence of mental illness and suicide in the Aboriginal population reflects the severe disruption of their society since European immigration. It reflects a context of emotional and social deprivation, characterised by trauma, loss and grief, the separation of families from their children, and the loss of their land, culture and identity. It is exacerbated by the impact of social inequity, stigma and racism in the post-colonial period (Parker, 2010).

\section{Intellectual disability among Aboriginal people}

The construct of intellectual disability is determined by what is seen as competent behaviour in a particular culture. In a Western culture, for example, small children may be considered intelligent if they are able to count up to ten or if they have a large vocabulary. In contrast, an indigenous child from a particular desert community in Australia may be expected to know directions, space and place or to demonstrate independence to be considered intelligent (Dingwall \& Cairney, 2010). In some Aboriginal cultures, people with intellectual disability were considered to have a specific impairment or 'sickness' that prevented them doing certain tasks, but they were still involved in the kinship system, with roles and responsibilities. Aboriginal families considered themselves to be accepting and supportive of family members who had a disability (Stopher \& D'Antoine, 2008).

The 2002 National Aboriginal and Torres Strait Islander Social Survey (Australian Bureau of Statistics, 2004) showed that $7 \%$ of the Aboriginal population aged over 15 years had an intellectual disability, compared with $1-2 \%$ of all Australians (Wen, 1997). As this figure was obtained by questioning family members, it is likely to be indicative of what is seen as intellectual disability by the Aboriginal community rather than being equivalent to disability measured by Western standards. Although Aboriginal people comprise just 3.5\% of the population of Western Australia, they represent $7.4 \%$ of all people registered with intellectual disability services (Glasson et al, 2005). It is important to note that tests used in the assessment of general intelligence are rarely culture-free. Accordingly, test bias could have contributed to the over-representation of indigenous Australians among those considered to have intellectual disability. Dingwall \& Cairney (2010) comment that the Queensland Test, which was developed in the 1960s and adapted for the purpose of evaluating the intelligence of indigenous peoples, is probably neither culture free nor an adequate measure of general intellectual ability. There are, though, currently no alternative tests that possess greater validity.

Potential causes of increased levels of intellectual disability among the Aboriginal population include intrauterine exposure to toxins such as alcohol, tobacco and cannabis, which could adversely affect fetal brain development. A lack of adequate antenatal and perinatal care is an issue in some remote communities. Prematurity, particularly when associated with poor intrauterine growth, often leads to delayed development. Postnatal factors such as trauma and infections also increase vulnerability to the later development of intellectual disability. In summary, the interplay of prenatal and environmental factors is likely to contribute to high rates of intellectual disability in the Aboriginal communities, although much work needs to be done to delineate the specific mechanisms further.

\section{Implications for psychiatric training}

The training of psychiatrists who will be working with Aboriginal people requires a multifaceted approach. Their education and training need to focus strongly on cultural awareness. They have to understand the prejudice that leads to discrimination, reflected by the interface of Aboriginality and disability. Such prejudice can militate against Aboriginal people receiving appropriate psychiatric diagnosis, treatment and care. Psychiatrists need to be aware of the important cultural differences between themselves and an Aboriginal person and the need for respect, adaptation and self-reflection.

In working with Aboriginal communities, psychiatrists need to take a culturally sensitive perspective on human development, and to understand that the presentation of disability and cultural responses to disability may be different. Assessment should be systemic, taking into consideration individual family and community factors. It is necessary for family and respected community members to be consulted, in order to determine whether the symptoms an individual is experiencing are explicable within a cultural context. The assessment should be undertaken in collaboration with Aboriginal mental health workers or cultural consultants.

It is important for psychiatrists working with Aboriginal peoples to have received training in developmental psychiatry. 
There is a high prevalence of conditions such as autismspectrum disorders and people with intellectual disability are strongly represented (Bhaumik et al, 2008). Training should incorporate a culturally appropriate interpretation of the mental state examination. For instance, lack of direct eye contact or social reciprocity in an Aboriginal setting is not necessarily evidence of impaired social functioning.

The establishment and retention of a specialised workforce in intellectual disability also needs to be urgently undertaken. For example, the Kimberley region of Western Australia, which spans $500000 \mathrm{~km}^{2}$ and has almost 25\% of the state's Aboriginal population, has just two adult psychiatrists. Neither has subspecialty training in intellectual disability. Although the Royal Australian and New Zealand College of Psychiatrists provides a 12-month training fellowship in intellectual disability and mental health, the programme does not specifically address Aboriginal intellectual disability. Forming links with groups of psychiatrists such as the Psychiatry of Learning Disability Faculty of the Royal College of Psychiatrists could help with gaining skills to meet the needs of indigenous people with intellectual disability.

\section{References}

Australian Bureau of Statistics (2004) National Aboriginal and Torres Strait Islander Health Survey, 2002. ABS.
Australian Bureau of Statistics (2006) National Aboriginal and Torres Strait Islander Health Survey, 2004-05. ABS.

Australian Health Ministers' Advisory Council (2006) Aboriginal and Torres Strait Islander Health Performance Framework Report. AHMAC.

Bhaumik, S., Tyrer, F. C., McGrother, C., et al (2008) Psychiatric service use and psychiatric disorders in adults with intellectual disability. Journal of Intellectual Disability Research, 52, 986-995.

Cohen, A. (1999) The Mental Health of Aboriginal Peoples - An International Review. World Health Organization.

Dingwall, K. M. \& Cairney, S. (2010) Psychological and cognitive assessment of Indigenous Australians. Australian and New Zealand Journal of Psychiatry, 44, 20-30.

Glasson, E. J., Sullivan, S. G., Hussain, R., et al (2005) An assessment of intellectual disability among Aboriginal Australians. Journal of Intellectual Disability Research, 49, 626-634.

Morgan, D. L., Slade, M. D. \& Morgan, C. M. A. (1997) Aboriginal philosophy and its impact on health outcomes. Australian and New Zealand Journal of Public Health, 1, 597-601.

Parker, R. (2010) Australia's Aboriginal population and mental health. Journal of Nervous and Mental Disease, 198, 3-7.

Stopher, K. \& D'Antoine, H. (2008) Aboriginal People with Disability: Unique Approaches to Unique Issues. Available at http://www. disability.wa.gov.au (last accessed March 2011).

Swan, P. \& Raphael, B. (1995) Ways Forward: National Consultancy Report on Aboriginal and Torres Strait Islander Mental Health. Office of Aboriginal and Torres Strait Islander Health.

Wen, X. (1997) The Definition and Prevalence of Intellectual Disability in Australia. AlHW Cat. No. DIS2. Australian Institute of Health and Welfare.

Zubrick, S., Silburn, S. R., Lawrence, D. M., et al (2005) Western Australian Aboriginal Child Health Survey: The Social and Emotional Wellbeing of Aboriginal Children and Young People. Curtin University of Technology and Telethon Institute for Child Health Research.

\title{
Services for adults with intellectual disability in Mexico: opinions and experiences of service users
}

\author{
Betania Allen-Leigh, ${ }^{1}$ Gregorio Katz $^{2}$ and Eduardo Lazcano-Ponce ${ }^{3}$
}

${ }^{1}$ Population Health Research Center, National Institute of Public Health, Cuernavaca, Morelos, Mexico; ${ }^{2}$ Integral Training and Development (CADI), Mexico State, Mexico; ${ }^{3}$ Population Health Research Center, National Institute of Public Health, Cuernavaca, Morelos, Mexico, email elazcano@insp.mx

\begin{abstract}
W e have conducted a qualitative study to collect information about services for adults and older adolescents with intellectual disability in Mexico, as part of ongoing research on intellectual disability from a public health and rights-oriented perspective. Our focus is on existing services and outstanding needs. One of our main goals is to provide an opportunity for people with intellectual disability to convey their experiences and express their opinions about the additional services they require. Three types of service are included: those supporting autonomy or independence (personal and economic);
\end{abstract}

those enhancing community inclusion; and those providing vocational opportunities.

\section{Method}

We interviewed a convenience sample of 17 young adults and older adolescents (9 women and 8 men, age range 16-41 years, median age 25 years) with moderate to mild intellectual disability, in central Mexico. Most of the people interviewed worked $(n=14)$ and most had used both public 